\title{
Real-time monitoring of microwave-promoted Suzuki coupling reactions using in-situ Raman spectroscopy
}

Nicholas E. Leadbeater* and Rebecca J. Smith

Department of Chemistry, University of Connecticut, 55 North Eagleville Road, Storrs, CT 06269-3060 USA

nicholas.leadbeater@uconn.edu

${ }^{1} \mathrm{H}$ and ${ }^{13} \mathrm{C}$ NMR data for the compounds prepared S4-14 


\section{Description of the apparatus:}

Microwave reactions were conducted using a modified monomode microwave unit (CEM Discover ${ }^{\circledR}$ ). The machine consists of a continuous Focused ${ }^{\mathrm{TM}}$ microwave power delivery system with operator selectable power output from 0$300 \mathrm{~W}$. Reactions were performed in glass vessels (capacity $10 \mathrm{~mL}$ ) sealed with a septum. The pressure is controlled by a load cell connected to the vessel via a non-invasive pressure measurement device above the septum surface. The temperature of the contents of the vessel was monitored using a calibrated infrared temperature control mounted under the reaction vessel. All experiments were performed using a stirring option whereby the contents of the vessel are stirred by means of a rotating magnetic plate located below the floor of the microwave cavity and a Teflon-coated magnetic stir bar in the vessel.

The Raman system was provided by Enwave Optronics (www.enwaveopt.com).

EXCITATION SOURCE: $\quad$ NIR, frequency stabilized, narrow linewidth diode laser at 785 nm. Laser power at sample $200 \mathrm{~mW}$. Linewidth $<2 \mathrm{~cm}^{-1}$. Fiber-coupled laser output $(100 \mu \mathrm{m}, 0.22 \mathrm{NA})$.

FIBER-OPTIC PROBE: $\quad$ Permanently-aligned two single fiber combination $100 \mu \mathrm{m}$ excitation fiber, $200 \mu \mathrm{m}$ collection fiber (0.22 NA). Working distance: $8 \mathrm{~mm}$ (standard). Rayleigh rejection: O.D. $>7$ at laser wavelength.

CCD DETECTOR: $\quad$ High sensitivity linear CCD array. Temperature regulated (at $13^{\circ} \mathrm{C}$ ) operation for long integration time and stable dark reference subtraction. Pixel Size:14 $\mu \mathrm{m} \times 200$ $\mu \mathrm{m}$ (2048 Pixels);16 Bit digitization.

SPECTROGRAPH: $\quad$ Symmetrical crossed Czerny-Turner design. Resolution: $\sim 10 \mathrm{~cm}^{-1}$ at $785 \mathrm{~nm}$. Excitation spectral coverage: $300 \mathrm{~cm}^{-1}$ to $2400 \mathrm{~cm}^{-1}$. Built-in software calibration.

SYSTEM SOFTWARE: $\quad$ Data collection software, data files exported into .dat format, converted to .txt and imported into Microsoft Excel.

To interface the microwave unit and Raman spectrometer, a hole $(0.8 \mathrm{~cm}$ i.d.) was drilled in the microwave cavity and an RF stub attached to the outer cavity wall (to prevent microwave leakage) and an extender (2.16 $\mathrm{cm}$ i.d.) attached to this, reaching through to the outer casing of the microwave unit. The fiber-optic probe was placed into the cavity and the laser focused through a quartz light tube. A distance between the reaction vessel and fiber-optic probe tip of $0.5 \mathrm{~cm}$ was optimal. 
Photographs of the apparatus:
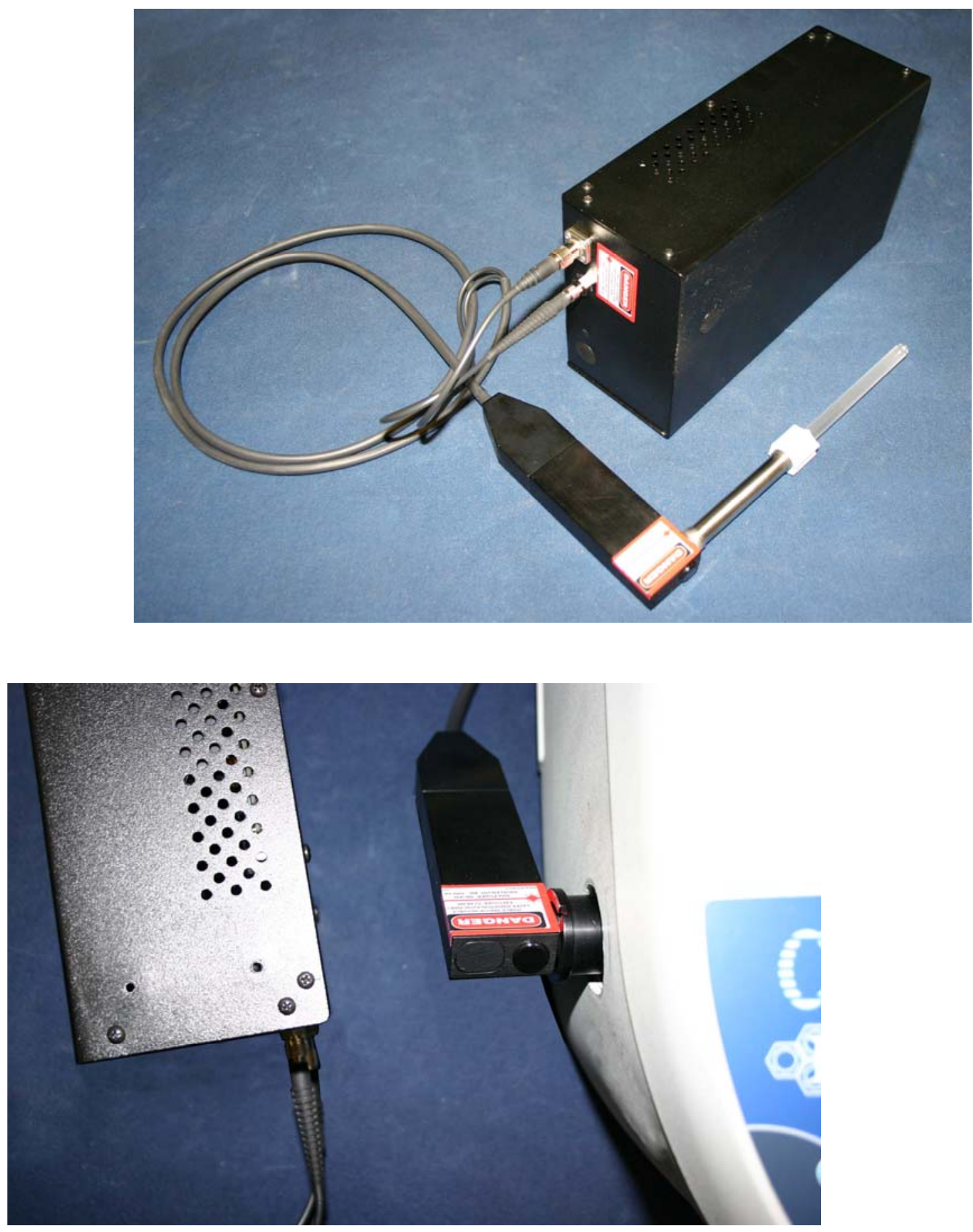


\section{General experimental:}

Unless noted otherwise, all materials were obtained from commercial suppliers and used without further purification. All reactions were carried out in air. ${ }^{1} \mathrm{H}$ - and ${ }^{13} \mathrm{C}-\mathrm{NMR}$ spectra were recorded at $293 \mathrm{~K}$ on a 300 or 400 $\mathrm{MHz}$ spectrometer.

\section{Typical experimental procedure:}

In a $10 \mathrm{~mL}$ glass tube was placed aryl bromide $(0.5 \mathrm{mmol})$, phenylboronic acid $(0.061 \mathrm{~g}, 0.5 \mathrm{mmol}), \mathrm{DBU}(0.075 \mathrm{~mL}$, $0.5 \mathrm{mmol})$, palladium acetate $(1 \mathrm{mg}, 0.8 \mathrm{~mol} \%)$, ethanol $(2 \mathrm{~mL})$, water $(1 \mathrm{~mL})$ and magnetic stir bar. The vessel was placed into the microwave cavity and sealed with a pressure lock. The microwave source was then turned on and microwave irradiation of $50 \mathrm{~W}$ was used to ramp the temperature from r.t. to $150{ }^{\circ} \mathrm{C}$ where it was held for 10 min by modulating the microwave power Raman spectra were recorded approximately every 8 sec throughout the reaction. Both the microwave and the Raman apparatuses were started simultaneously. After allowing the reaction mixture to cool down to r.t., the vessel was opened and the contents poured into a separatory funnel. Water ( $25 \mathrm{~mL})$ and diethyl ether $(25 \mathrm{~mL})$ were added and the organic material extracted and removed. After further extraction of the aqueous layer with diethyl ether, combining the organic washings and drying them over $\mathrm{MgSO}_{4}$, the solvent was removed in vacuuo leaving the crude product.

4-Acetylbiphenyl: ${ }^{1} \mathrm{H}$ NMR $\left(\mathrm{CDCl}_{3}\right) \delta 8.06(\mathrm{~d}, \mathrm{~J}=8.4 \mathrm{~Hz}, 2 \mathrm{H}), 7.70(\mathrm{~d}, \mathrm{~J}=8.4 \mathrm{~Hz}, 2 \mathrm{H}), 7.67(\mathrm{~d}, \mathrm{~J}=7.5 \mathrm{~Hz}, 2 \mathrm{H}), 7.50(\mathrm{~d}, \mathrm{~J}$ $=7.5 \mathrm{~Hz}, 2 \mathrm{H}), 7.45$ (d, J = 7.4 Hz, 1H), 2.66 (s, 3H); ${ }^{13} \mathrm{C} \mathrm{NMR}\left(\mathrm{CDCl}_{3}\right) \delta$ 197.76, 145.80, 139.90, 135.86, 128.97, 128.92, $128.24,127.28,127.23,26.70$.

4-Methylbiphenyl: ${ }^{1} \mathrm{H}$ NMR $\left(\mathrm{CDCl}_{3}\right) \delta 7.64(\mathrm{~d}, \mathrm{~J}=7.1 \mathrm{~Hz}, 2 \mathrm{H}), 7.55(\mathrm{~d}, \mathrm{~J}=8.1 \mathrm{~Hz}, 2 \mathrm{H}), 7.48(\mathrm{t}, \mathrm{J}=7.5 \mathrm{~Hz}, 2 \mathrm{H}), 7.37(\mathrm{t}, \mathrm{J}$ $=7.3 \mathrm{~Hz}, 1 \mathrm{H}), 7.30(\mathrm{~d}, \mathrm{~J}=7.9 \mathrm{~Hz}, 2 \mathrm{H}), 2.45$ (s, 3H) ); ${ }^{33} \mathrm{C} \mathrm{NMR}\left(\mathrm{CDCl}_{3}\right) \delta$ 141.21, 138.40, 137.04, 129.51, 128.74, 127.02, $127.00,21.14$

4-Methoxybiphenyl: ${ }^{1} \mathrm{H}$ NMR $\left(\mathrm{CDCl}_{3}\right) \delta 7.53-7.58(\mathrm{~m}, 4 \mathrm{H}), 7.43(\mathrm{t}, \mathrm{J}=7.7 \mathrm{~Hz}, 2 \mathrm{H}), 7.32(\mathrm{t}, \mathrm{J}=7.4 \mathrm{~Hz}, 1 \mathrm{H}), 6.99(\mathrm{~d}, \mathrm{~J}=$ $8.8 \mathrm{~Hz}, 2 \mathrm{H}), 3.86(\mathrm{~s}, 3 \mathrm{H}) ;{ }^{13} \mathrm{C} \mathrm{NMR}\left(\mathrm{CDCl}_{3}\right) \delta 159.17,140.85,133.80,128.72,128.16,126.75,126.66,114.22,55.35$.

2-Methylbiphenyl: ${ }^{1} \mathrm{H}$ NMR $\left(\mathrm{CDCl}_{3}\right) \delta 7.45(\mathrm{t}, \mathrm{J}=7.3 \mathrm{~Hz}, 2 \mathrm{H}), 7.35-7.39(\mathrm{~m}, 3 \mathrm{H}), 7.27-7.30(\mathrm{~m}, 4 \mathrm{H}), 2.31(\mathrm{~s}, 3 \mathrm{H}) ;{ }^{13} \mathrm{C}$ NMR (500 MHz, $\left.\mathrm{CDCl}_{3}\right) \delta$ 142.0, 135.4, 130.3, 129.8, 129.2, 128.0, 127.3, 126.8, 125.8, 20.5.

4-Cyanobiphenyl: ${ }^{1} \mathrm{H}$ NMR $\left(\mathrm{CDCl}_{3}\right) \delta 7.75(\mathrm{~d}, \mathrm{~J}=8.5 \mathrm{~Hz}, 2 \mathrm{H}), 7.71(\mathrm{~d}, \mathrm{~J}=8.5 \mathrm{~Hz}, 2 \mathrm{H}), 7.62(\mathrm{~d}, \mathrm{~J}=7.7 \mathrm{~Hz}, 2 \mathrm{H}), 7.52(\mathrm{t}, \mathrm{J}$ $=7.5 \mathrm{~Hz}, 2 \mathrm{H}), 7.46(\mathrm{t}, \mathrm{J}=7.4 \mathrm{~Hz}, 1 \mathrm{H}) ;{ }^{13} \mathrm{C} \mathrm{NMR}\left(\mathrm{CDCl}_{3}\right) \delta 145.7,139.2,132.6,129.1,128.7,127.8,127.3,119.0,110.9$. 


\section{4-Acetylbiphenyl}

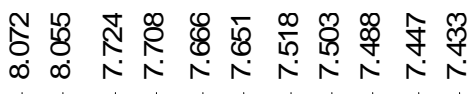

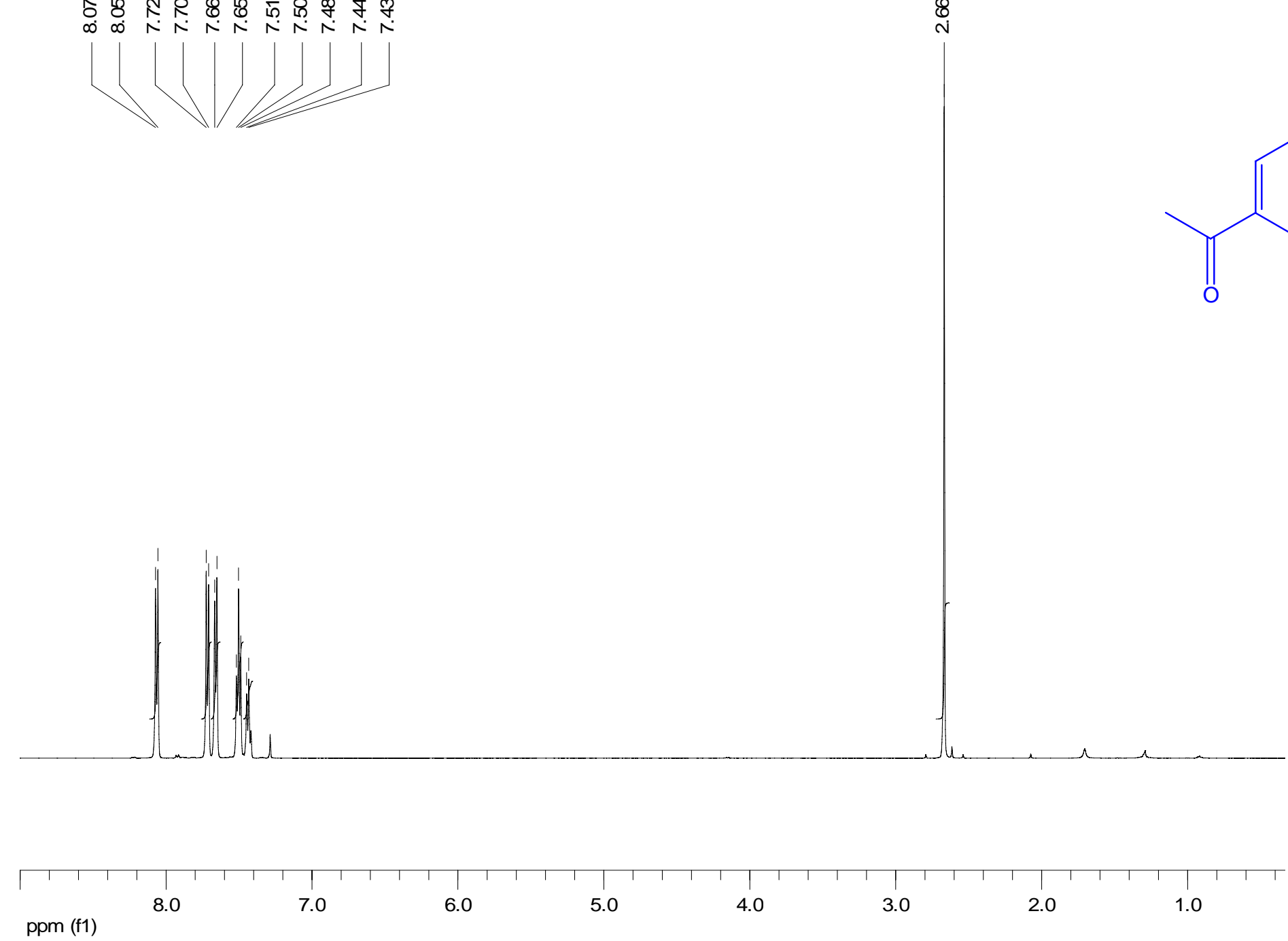




\section{4-Acetylbiphenyl}
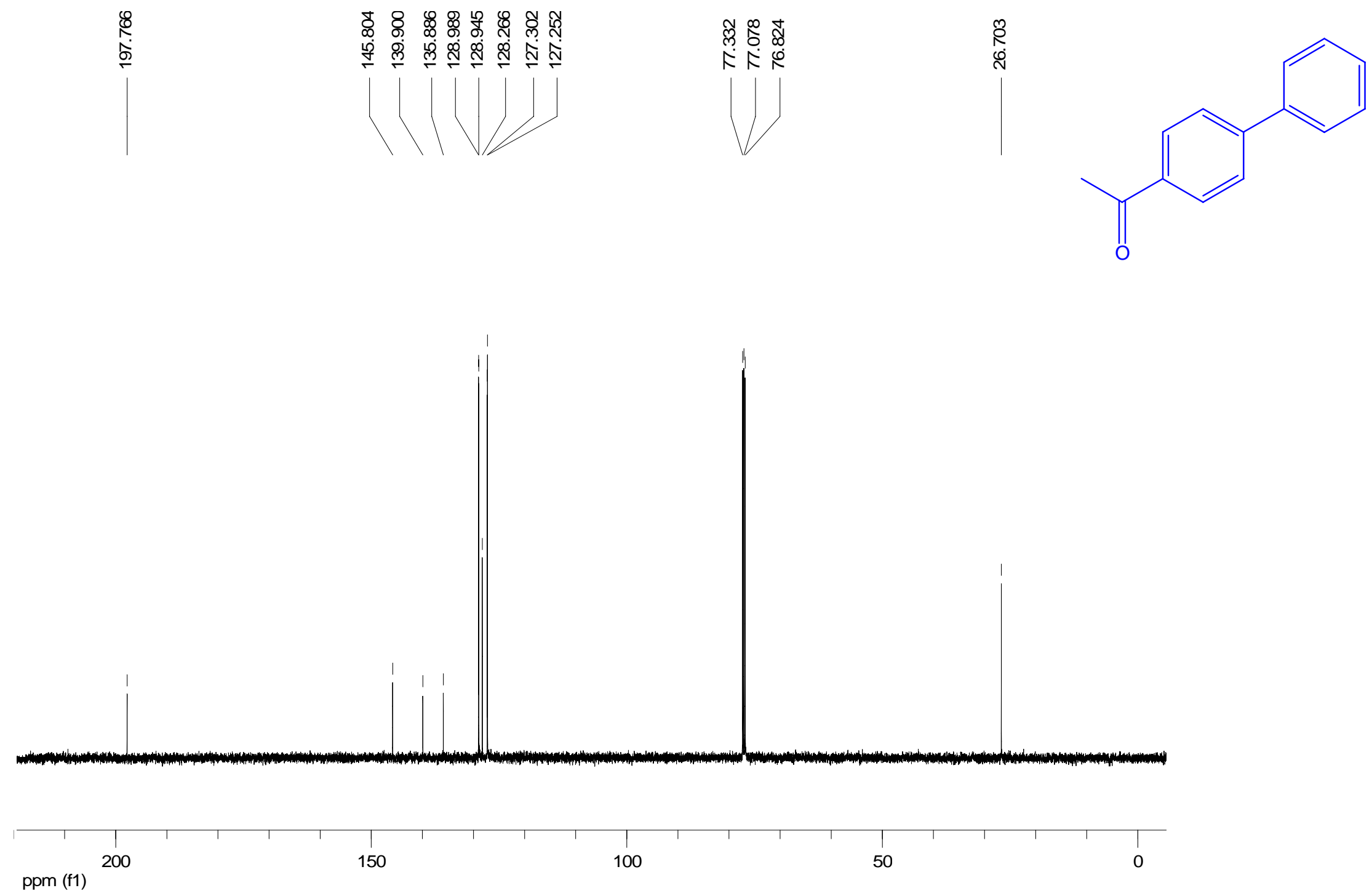


\section{4-Methylbiphenyl}

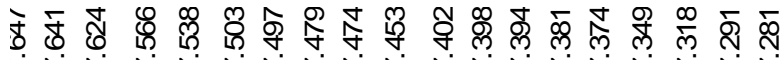
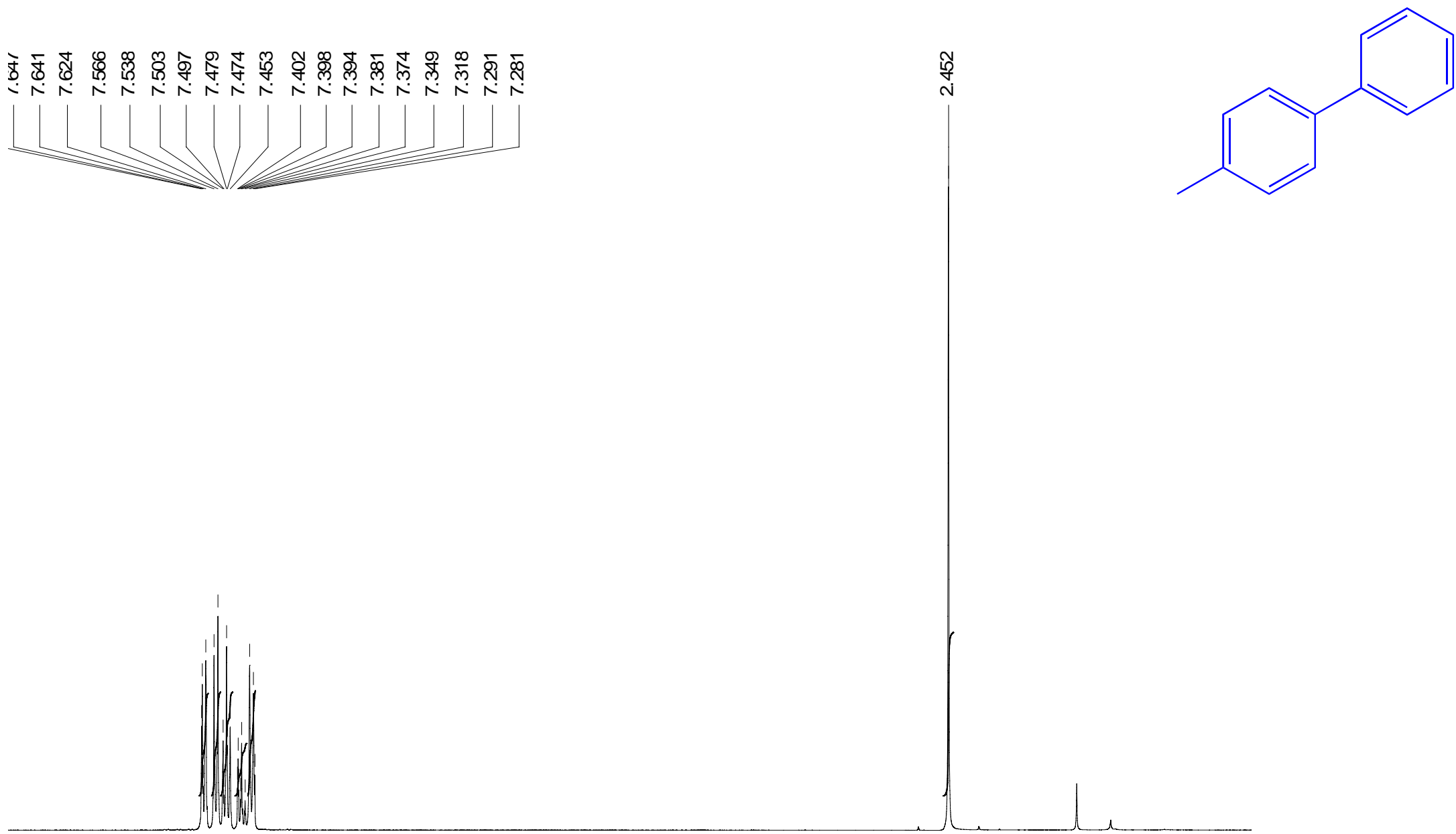

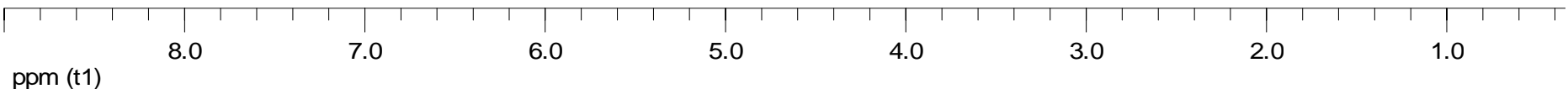




\section{4-Methylbiphenyl}

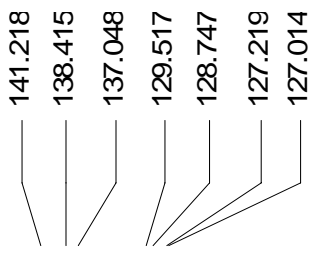

过 娄

К $\hat{N}$
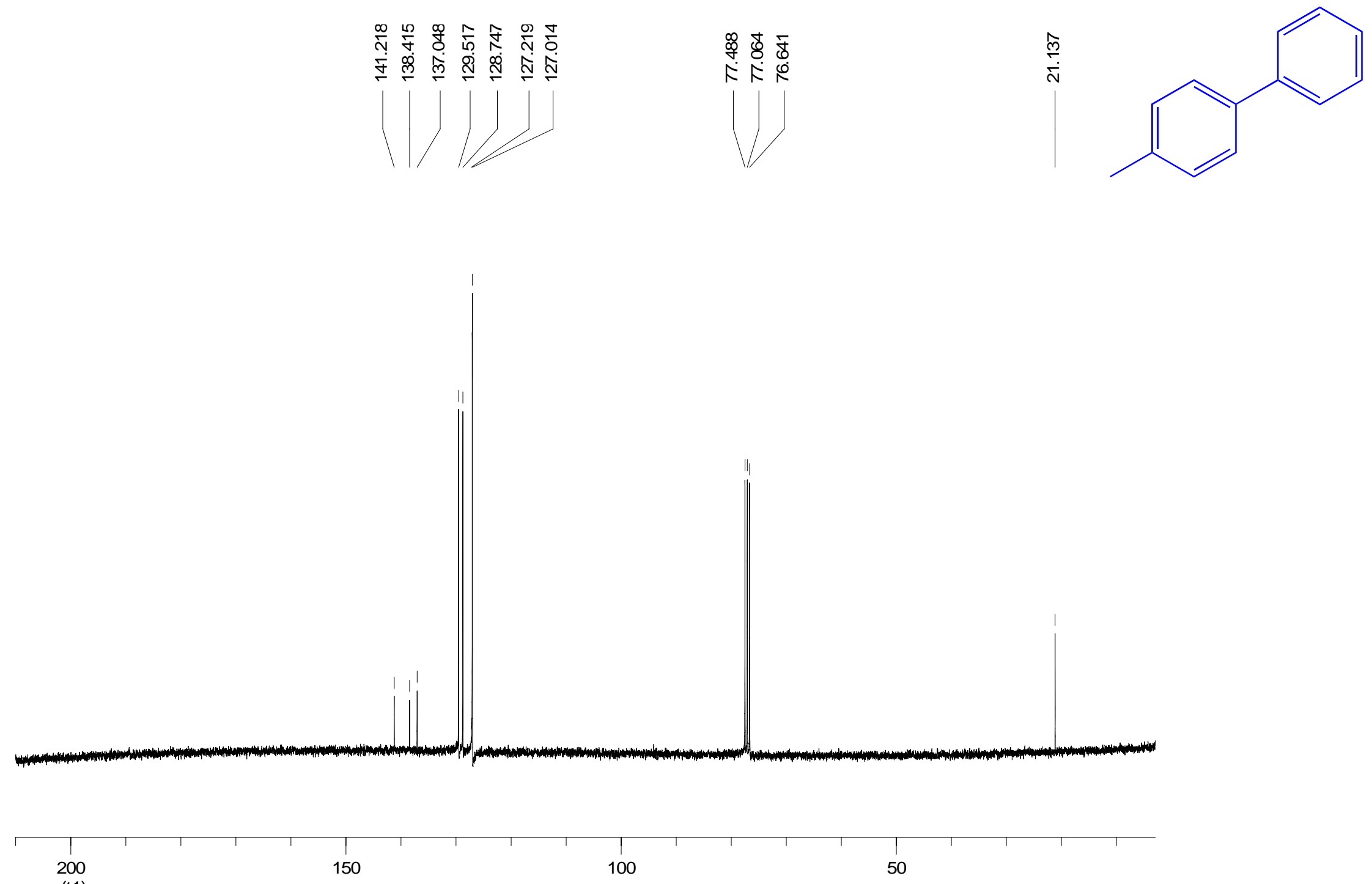

ppm (t1) 


\section{4-Methoxybiphenyl}

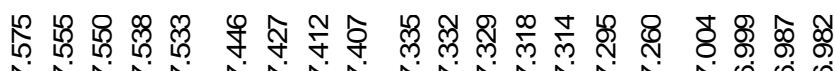

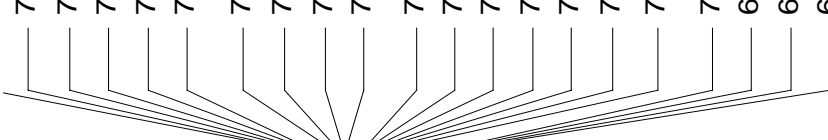

๕̊
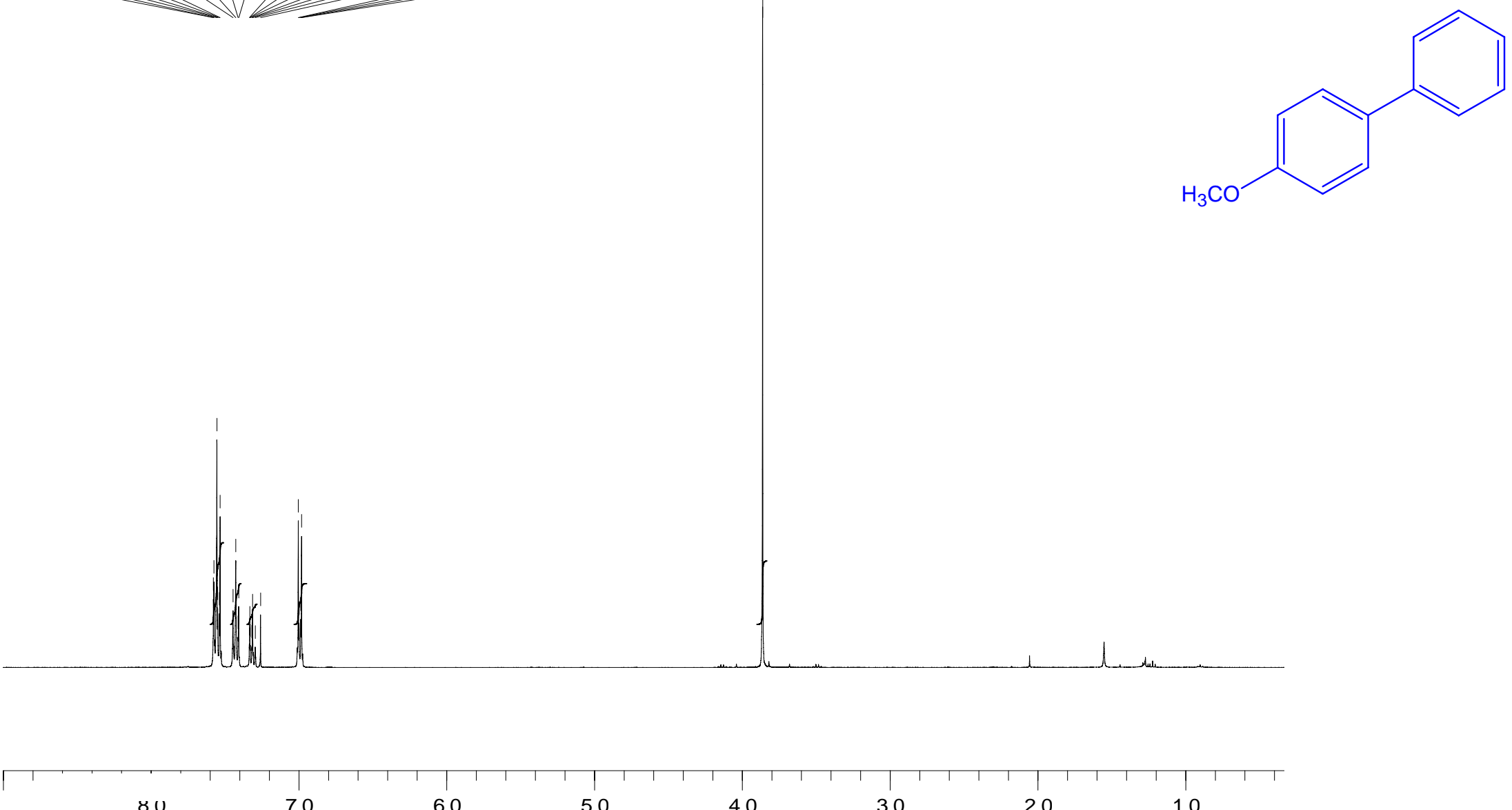

४.U

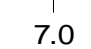

6.0

5.0

4.0

3.0

2.0

ppm (t1) 


\section{4-Methoxybiphenyl}
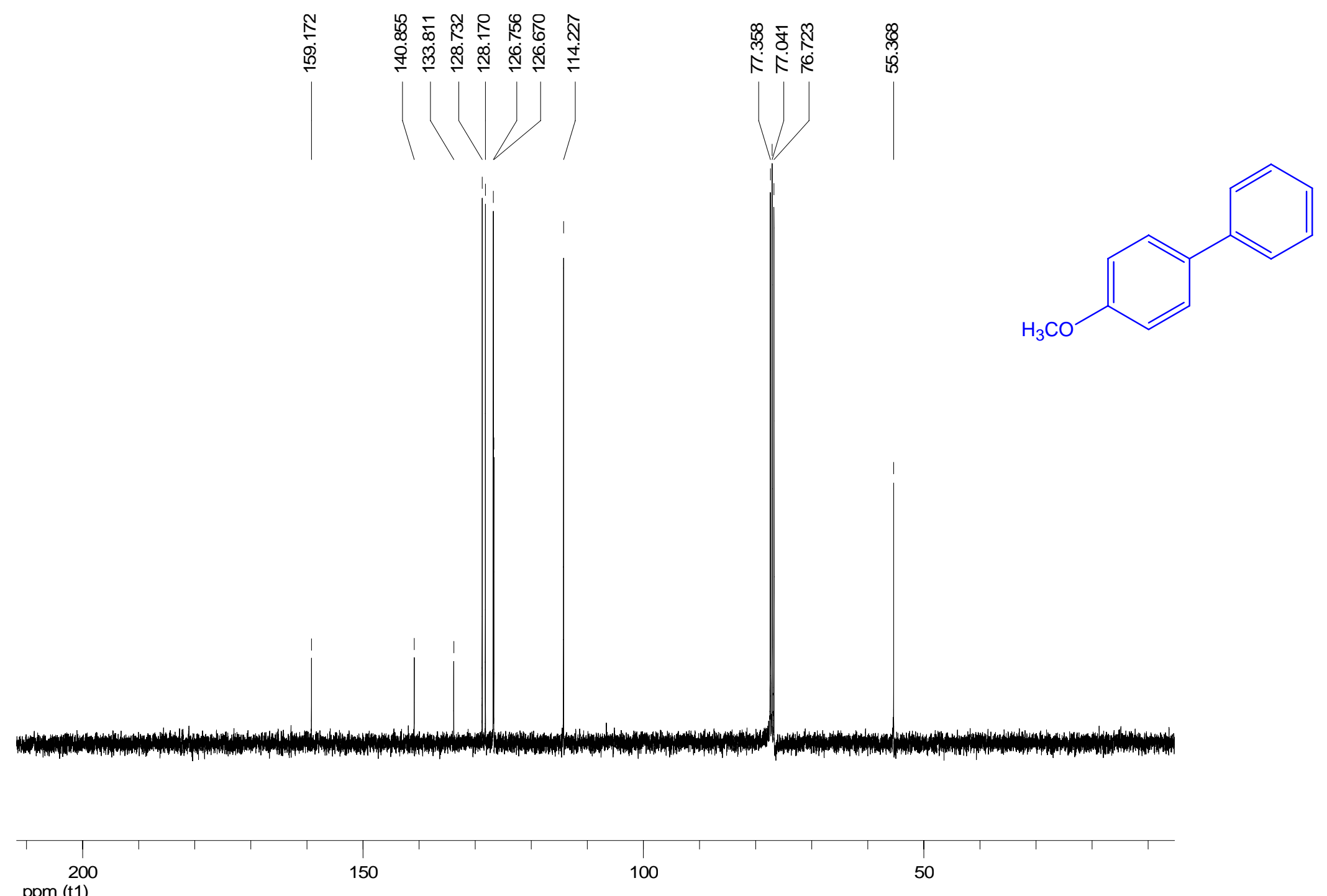


\section{4-Cyanobiphenyl}

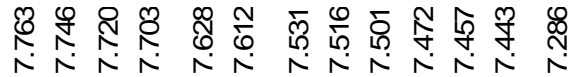

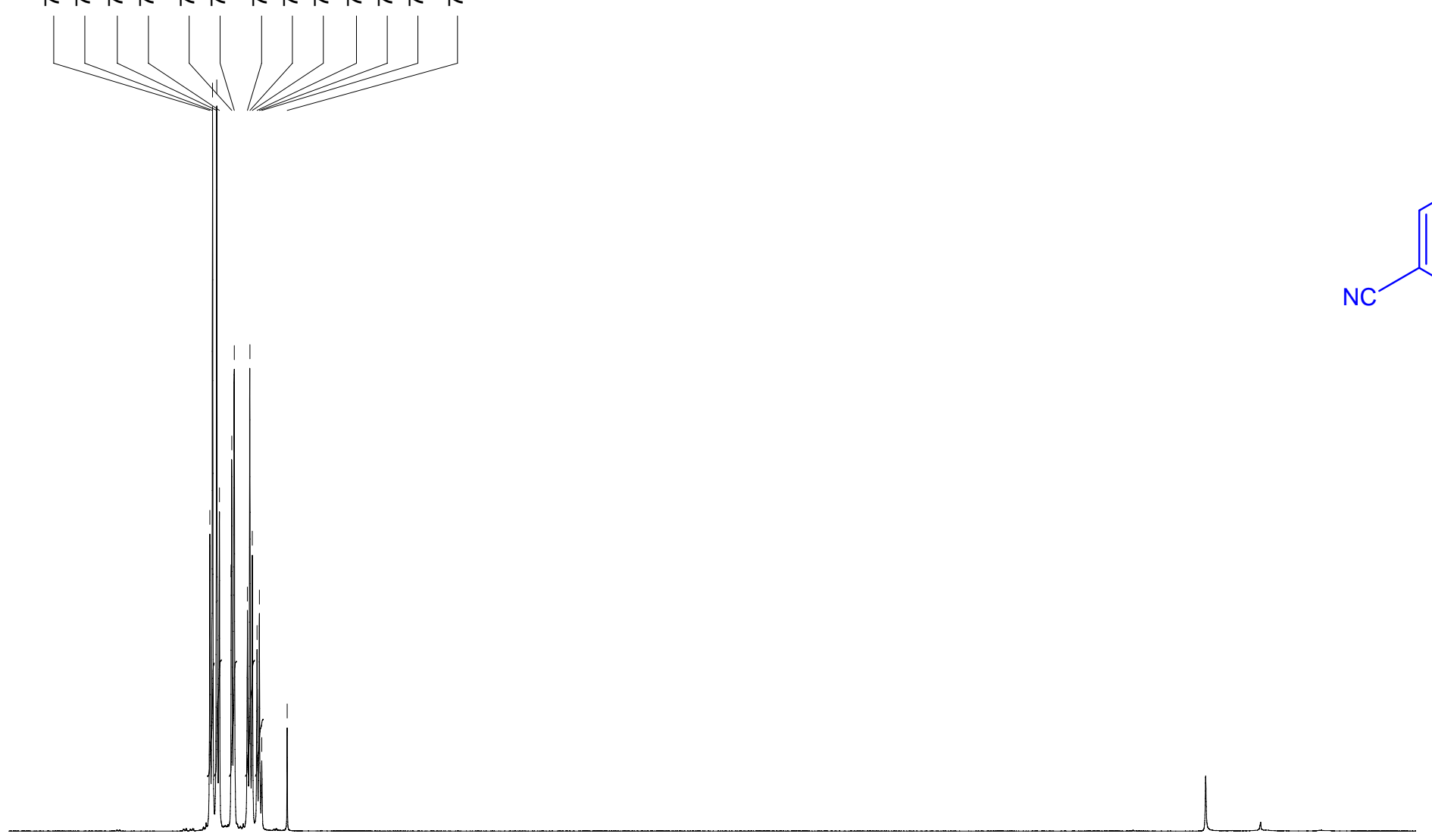

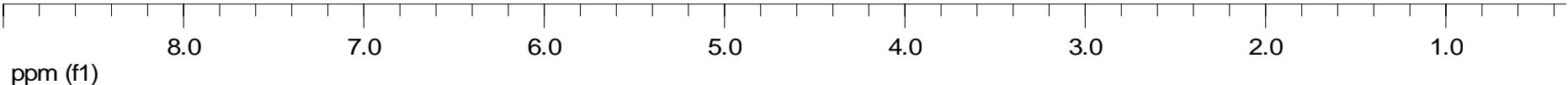




\section{4-Cyanobiphenyl}
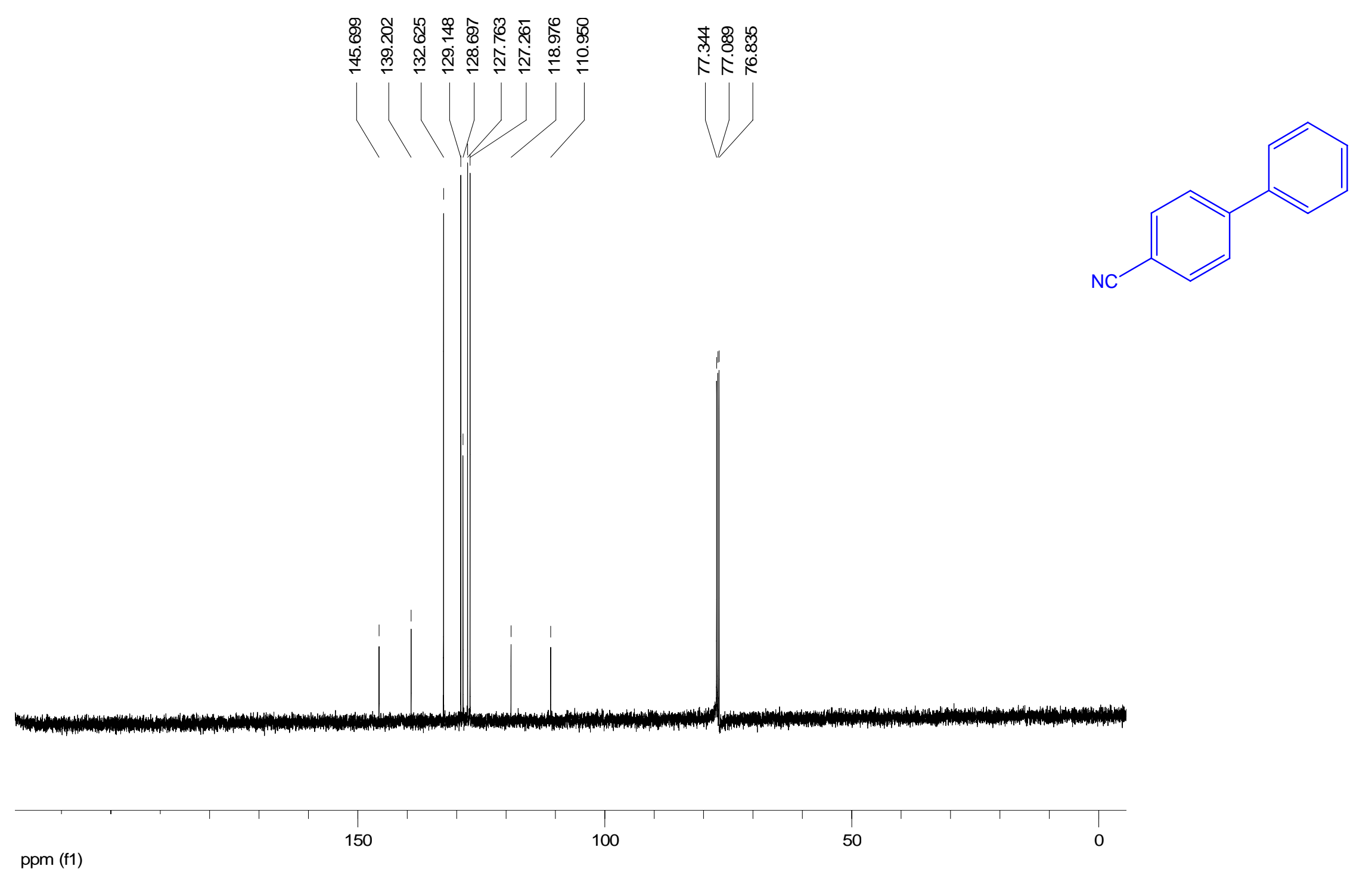


\section{2-Methoxybiphenyl}

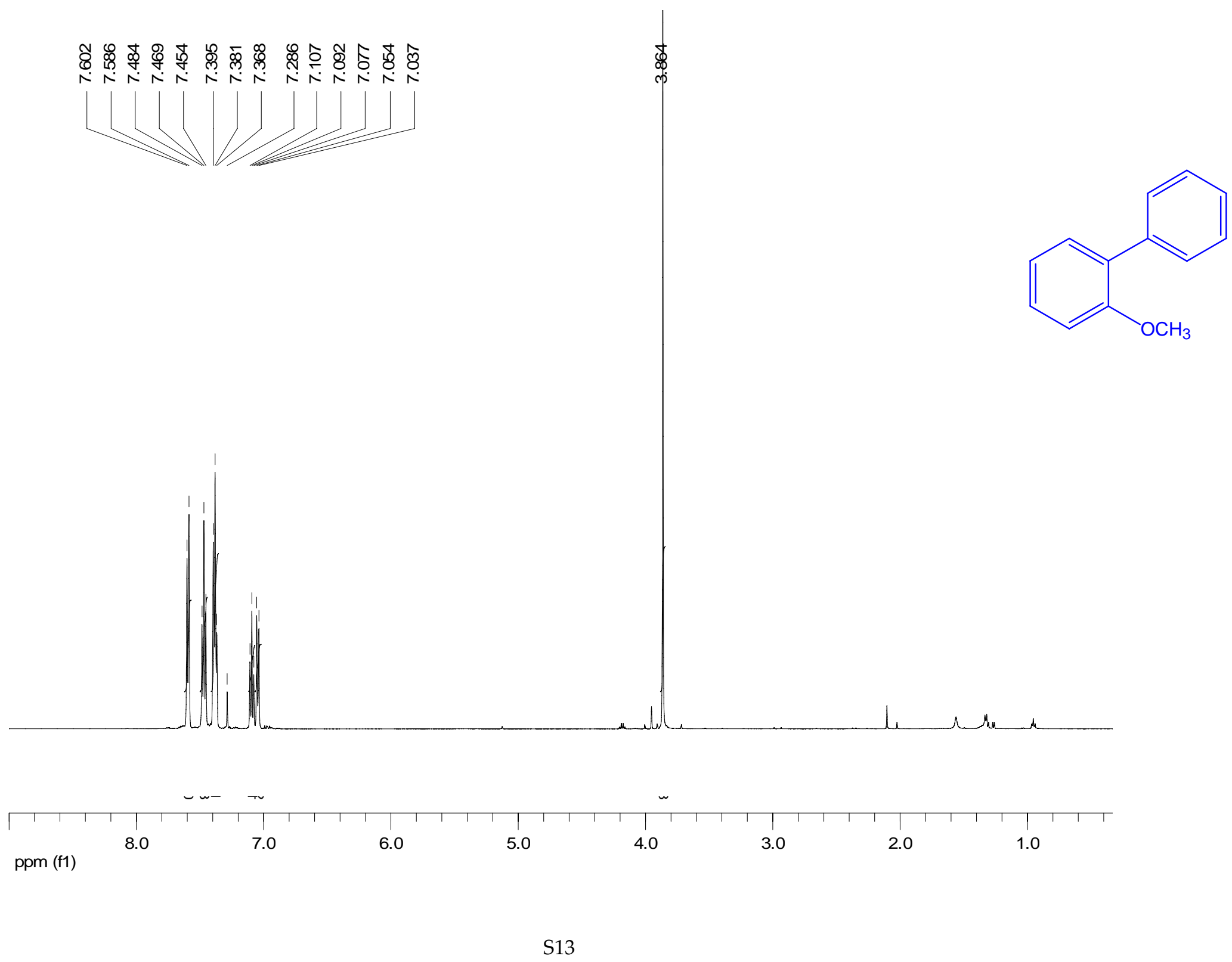




\section{2-Methoxybiphenyl}

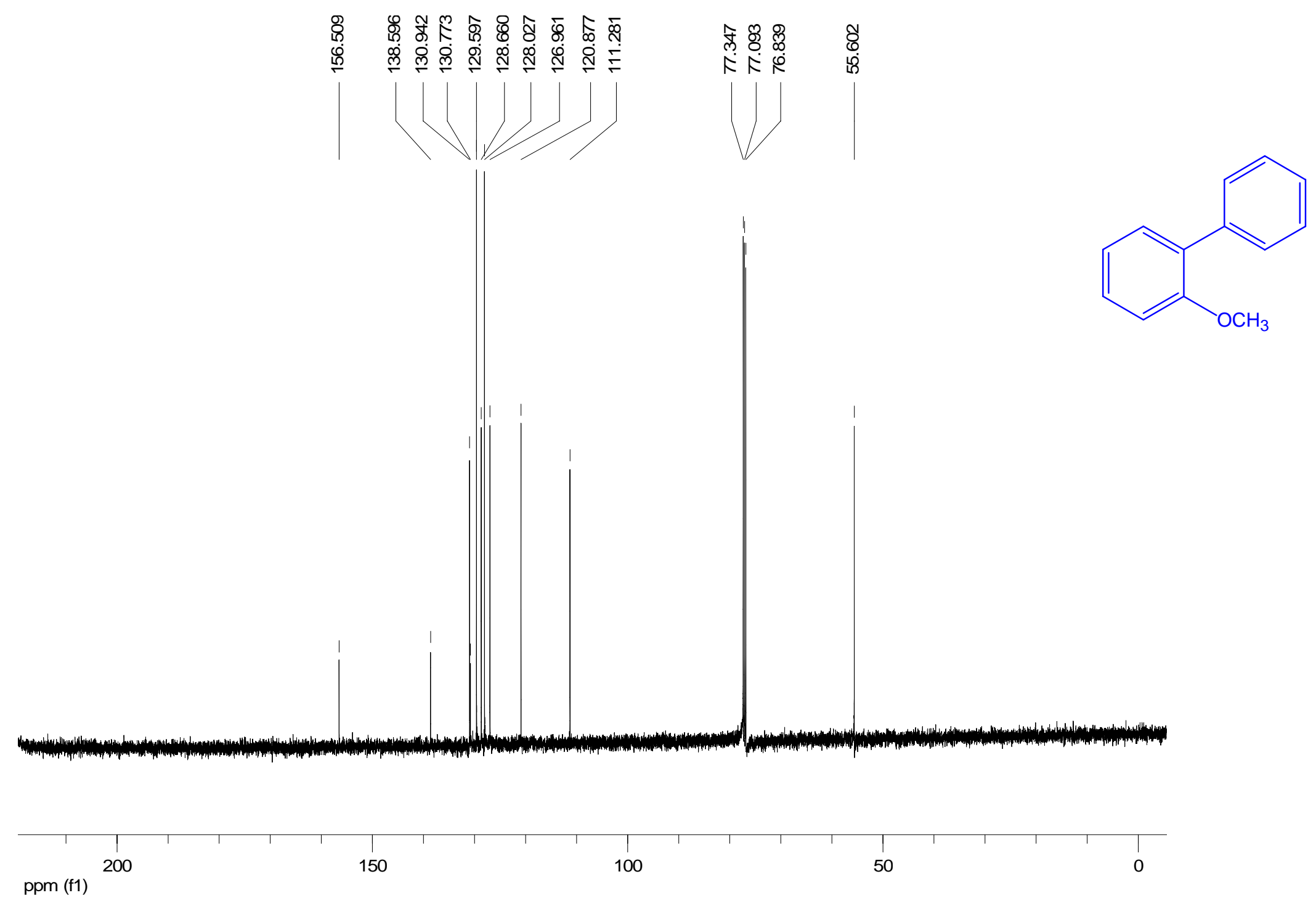

